\title{
Distributed Brillouin Sensors for Simultaneous Temperature and Strain Sensing
}

\author{
Ilie Popa ${ }^{1 *}$, Alexandru-Alin Jderu ${ }^{2,3}$, Cristian Livede ${ }^{2,3}$, Dorel Dorobanțu², \\ Marius Enachescu ${ }^{2}$ \\ ${ }^{1}$ University of Pitesti, Electronics, Computers and Electrical Engineering \\ Department, Pitesti, Arges, 110040, Romania \\ ${ }^{2}$ Center for Surface Science and Nanotechnology (CSSNT), \\ University Politehnica of Bucharest, Romania \\ ${ }^{3}$ Sc NanaoPro Start MC Srl, Pitesti, Arges, 110310, Romania \\ *ci.popa@yahoo.com
}

\begin{abstract}
This work makes an overview of Distribute Brillouin Sensors (DBS) with Optical Fiber (OF) aiming specifically at their applications for the temperatures and strain measuring in different structures. Firstly, Brillouin Scattering (BS) effect of light waves through OF, Stimulated Brillouin scattering (SBS) Stokes and anti-Stokes are described. It continues with the DBS networks presentation, the studied methods and techniques, inventorying their main practical applications. It ends with a comparative presentation of the basic parameters for the presented different techniques followed by conclusions.
\end{abstract}

\section{Introduction}

Temperature and strain material measurement using DBS is based on BS effect discovered by Léon Brillouin. BS occurs when light transmitted through a transparent material interacts with the propagation time and space generating periodic refractive index $(\mathrm{RI})$ variations due to density variation material. RI of a transparent material changes when the material is deformed by compressionexpansion, bending or temperature variations.

The interaction result between the light beam and the propagation material strain lies in the fact that a fraction of the light wave which propagates, changes its frequency and energy along the preferential angles.

If the transport material of light wave is a solid crystal, a chain macromolecular condensed or a viscous liquid, the connections between the atomic frequency and the transport material strain, represented as consisting of quasiparticle, could be: the acoustic oscillation of material mass (called phonons); the electrical charges material displacement (called polarons, in dielectric); oscillating magnetic spin (called magnons, in magnetic materials).

From the solid physics perspective, BS is given by an interaction between an electromagnetic wave and one of three crystalline material quasiparticles mentioned above. Scattering is inelastic: the photon can lose energy (Stokes process) to create one of the three types of quasiparticles, or gain energy (anti-Stokes process) by absorbing one of them. A change of the photon energy corresponds to a Brillouin frequency shift (BFS) proportional to the energy released/absorbed by quasiparticle. Thus, the BS can be used to measure the energy, the wavelength and oscillation frequency of different types of atomic chains.

$B S$ is a result of direct interaction between light waves and the elastic acoustic waves.

Considering that the incident light rays frequency is $v$, and wavelength is $\lambda(\lambda=c / v$, where $c$ is the light speed in the crystal) are reflected by a front of plane acoustic waves, their direction changing with angle $\theta$ as shown in Figure 1 .

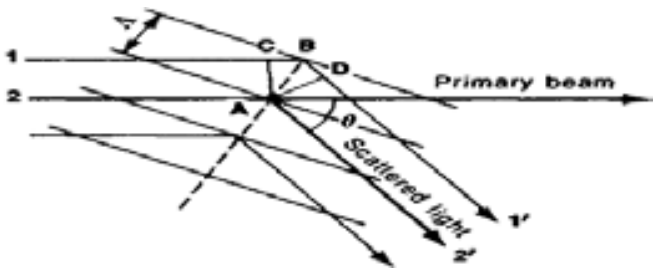

Figure 1. $\lambda_{b}$ is Brillouin scattering wavelength.

In order to get a reflected rays maximum intensity in an interference direction, the optical distance $\mathrm{CB}+\mathrm{DB}$ between rays 1-1' and 2-2', reflected by the adjacent wave fronts, must be equal to $\lambda[1]$ :

$$
2 \Lambda \cdot \sin \frac{\theta}{2}=2 n \lambda_{a} \sin \frac{\theta}{2}=\lambda=\frac{1}{v}
$$


where: $\Lambda=A B$ is the length of the scattering elastic (hypersonic) wave; $n$ is RI of the OF core; $\lambda_{a}$ is acoustic wavelength.

Light waves reflection by acoustic waves is equivalent to the incident light wave modulation with the frequency sound waves.

The frequency shift of light waves $\mathrm{BS}, v_{b}$, is equivalent to the acoustic waves frequency, $v_{a}$, as expressed in:

$$
v_{b}(T, \varepsilon)=\left.\frac{2 n}{\lambda} v_{a}\right|_{\theta=180^{\circ}}
$$

which depends on the acoustic wave velocity in the material which, in its turn, is dependent on the temperature, $T$, and the relative strait, $\varepsilon$. It is considered, $\theta=180^{\circ}$, because the maximum sensitivity of the sensor network is obtained when the scattered optical light stream is back-propagated. The light scattered frequency relative shift is:

$$
\frac{\Delta v}{v}= \pm \frac{2 v_{a}}{c \cdot \sin \frac{\theta}{2}}
$$

where, $\Delta v=v_{b}-v$ is the width of the scattering or amplification Brillouin.

Although the light frequency variation values of $\mathrm{BS}, \Delta v$ are relatively small, they can be acceptably measured by interferometry.

The fundamental parameters used in measurements of $T$ and $\varepsilon$ distribution along the OF are: the $B F S$ and the power exchange (the Brillouin signal power). For this, the networks of the optical DBS were developed.

The development of lasers has improved, not only the possibilities observation BS, but also, led to the discovery of Stimulated Brillouin Scattering, which is distinguished by high intensity and more features quality enhanced.

Spontaneous BS is determined by the density fluctuations caused by the acoustic waves pressure propagated in OF, the acoustic waves being generated by the molecular thermal agitation. The light frequency is Doppler shifted depending on the speed of acoustic waves in the OF (several $\mathrm{GHz}$ shift in the glass). The spontaneous BS is very weak (30 dB weaker than Rayleigh scattering), SBS becoming of great importance.

In the case of intense radii (for example laser light) passing through OF, the light beam electric field variations may produce acoustic vibrations in the material by the electrostriction and the ray undergoes a BS due to these vibrations, usually in the opposite direction of the input beam phase. This process is known as Stimulated Brillouin Scattering (SBS). If the input wave power is greater than the minimum threshold, then SBS occurs and because the minimum threshold is approximately $5 \mathrm{~mW}$, it can be said that the $\mathrm{OF}$ SBS effect occurs at low power levels.

Any optical intensity dynamic change can induce a pressure wave by electrostriction. Such a model of intensity variation may result from interference of two light waves with different counter-propagated wavelengths in FO. In this case, an optical-beat signals within $\mathrm{OF}$ is generated. It creates a pressure wave having the same frequency with optical-beat signal, schematically showed in Figure 2. [2]

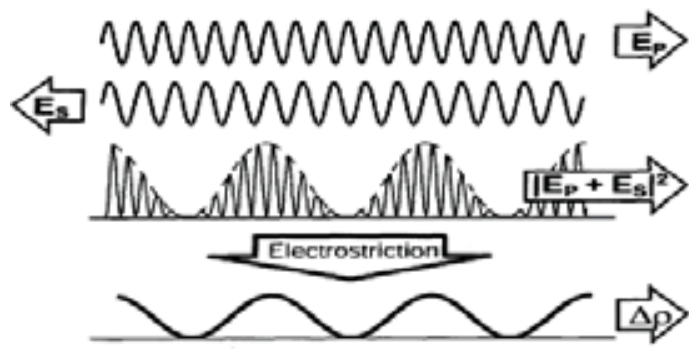

Figure 2. Generation of the pressure wave by electrostriction.

BS can be Stokes type when the wave frequency is shifted lower, and anti-Stokes, when the frequency is shifted higher, as showed in Figure 3 [2].

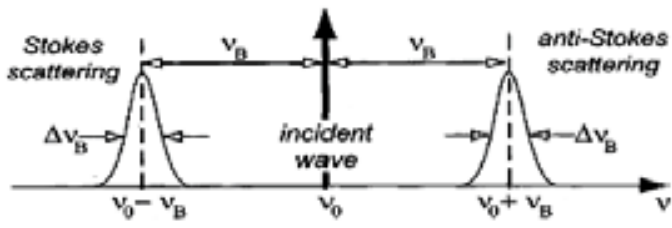

Figure 3. Typical Brillouin back-scattering radiation spectrum in OF. Important parameters are: the BFS $\left(v_{B}\right)$ and the Brillouin linewidth $\left(\Delta v_{B}\right)$.

\section{Distributed Brillouin Sensors for Simulta- neous Temperature and Strain Sensing}

\subsection{General information}

The DBS technology with OF is one of the most important areas of sensors OF that provides measurements of temperature, strain, vibrations etc., in industrial, military and civilian installations, 
with high resolution, on long distances [3]. A unique feature of OF distributed systems is their ability of continuous reading, almost in real time, the measured parameter being as a function of position, in any point along the fiber sensing, covering large infrastructures and distances [4].

The existing technologies use different approaches for distributed sensors, which practically, refer to the phenomenon of light scattering, interferometry, Bragg grid, and the optical loss in quasi-distributed sensors $[5,6]$.
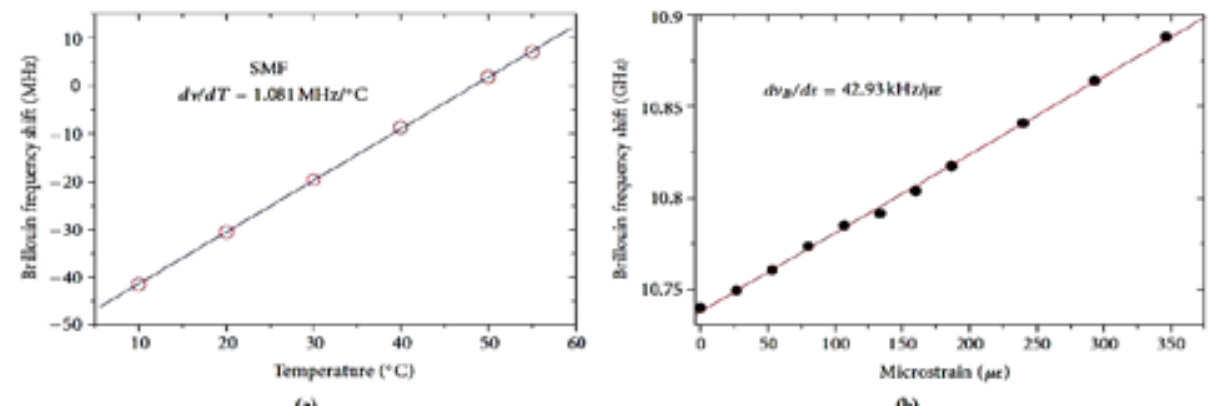

(b)

Figure 4. The BS frequency shift dependence on the temperature and strain. Experimental data.

Based on BS effect, are developed following configurations of the distributed fiber optic sensors:

1. Brillouin Optical Time-Domain Analysis

(BOTDA);

2. Brillouin Dynamic Grating Distributed Sensing

(BDG-DS);

3. Brillouin Optical Time-Domain Reflectometry

(BOTDR), OTDR based on spontaneous BS;

4. Brillouin Optical frequency-domain analysis

(BOFDA);

5. Brillouin Optical Correlation-Domain Analysis (BOCDA);

6. Brillouin Optical Correlation-Domain

Reflectometry (BOCDR);

7. Brillouin Echo Distributed Sensing (BEDS).

\subsection{Optical time-domain approach}

The time-domain approach for the BFS measuring in distributed OF is based on the use of a pulsed light beam (probe), which interacts with a continuous beam of light counter- propagated (CWpump). At any moment during the downward pulse propagation in the fiber, SBS effect occurs only in the fiber region where the two beams overlap. CW intensity received at the section where the pulse is launched is monitored as a function of time. This footprint time provides spatial information along
To analyze and estimate the detection performances are using the following parameters: spatial resolution; measurement accuracy; sensing range; measurement-acquisition time. These factors are, generally, linked together and a factor improving can lead to the deterioration of one or more of the others.

An example for the BS dependency of temperature and strain into a standard Single Mode Fiber (SMF) with BFS about 10 to $11 \mathrm{Ghz}$ at $1550 \mathrm{~nm}$ is showed in Figure 4 [7]. 
Figure 5 shows schematically the BS spectrum according to the BOTDA technique.

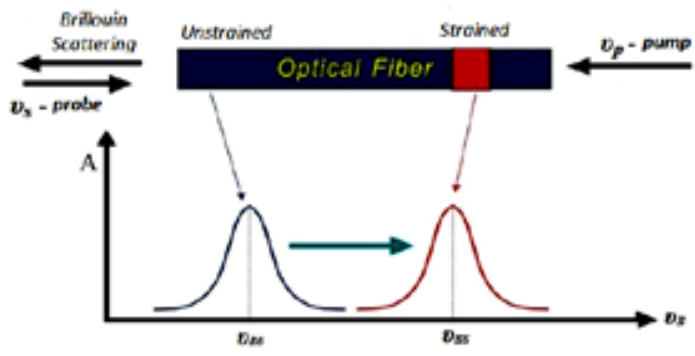

Figure 5. BS spectrum in BOTDA technique.

Measuring time in a traditional BOTDA system is of minutes order and it depends on the length of the OF. This is a serious disadvantage for dynamic monitoring of the structures health.

When the pulse was reduced to $1 \mathrm{~ns}$, which is equivalent to $10 \mathrm{~cm}$ resolution, the weak Brillouin signal, due to the short interaction, significantly reduced Signal/Noise Ratio (SNR), and therefore the strait and temperature achievable resolutions are reduced. To solve this problem, a differential pulse-width pair Brillouin optical time-domain analysis (DPP-BOTDA) for high spatial resolution sensing [9] was proposed. This method uses two separate different pulses: a long pulse (a few tens of nanoseconds) with a small pulse-width difference (a few nanoseconds) to map the Brillouin Gain Spectrum (BGS) of the sensing fiber. The differential BGS can be obtained by subtracting between the two BGSs, and its spatial resolution is determined by the pulse-width difference of the two separate long pulses. The DPP-BOTDA provides several advantages over conventional BOTDA: (1) Narrowband BGS (a few tens $\mathrm{MHz}$ ) and high spatial resolution (smaller than $1 \mathrm{~m}$ ) can be obtained simultaneously. (2) The differential BGS provides stronger signal intensity and thus better SNR than that of directly using the narrow pulse in BOTDA when the pulse-width difference of the two long pulses equals to the narrow pulse width.

\subsection{Brillouin Dynamic Grating Distributed Sensing}

Brillouin optical fibers sensitive grating (FBG) type are silicon fibers in which sensitive elements intrinsic form of grating (grill) are photo-engraved. Practically, a periodic structure is engraved in the OF core and this periodic structure will reflect a specific optical wavelength that depends on the periodicity [4]. Varying periodicity, the wavelength will vary, too. This period depends on the ambient temperature and strait and therefore it is the basis for a simple sensor (for example, a fiber grid) that can easily be interrogated.

The basic principle concerning the operation of the system FBG sensors is to monitor wavelength shift of the Bragg signal reflected by the measured changes. Bragg wavelength $\lambda_{B}$, or a grating resonance condition is expressed as:

$$
\lambda_{B}=2 n \Lambda
$$

where, $A$ is the periodicity of the grid and $n$ is the RI of the OF.

When such a device is illuminated by a broadband light source, a narrow band spectral component of the Bragg wavelength is reflected by the grating. This spectral component misses in the transmitted light. The bandwidth of the reflected signal depends on several parameters, including the length of the grid, but typically is about 0.05 $0.3 \mathrm{~nm}$ in most sensor applications. Disturbance (for example external deformation and temperature variations) of grid results in a shift of Bragg wavelength grid can be detected in each spectrum reflected or transmitted.

The grids can be: uniform, when the grid periodicity is uniform along the axis of the fiber core and the modulation degree of the RI is constant; non-uniform, when the grid periodicity is non-uniform along the axis of the core and the modulation degree of the RI need not be constant.

In more recent studies, the concept of the Brillouin dynamic gratings (BDG-DS) in singlemode fiber with the polarization maintaining (Differential Pulse-width Pairs - DPP) [10] was implemented. In this concept, acoustic waves generated in the SBS process by optical waves (pump waves) in a single polarization, are used for reflecting an orthogonal polarized wave (probe wave) at a different frequency from the frequency of the pump.

\subsection{Brillouin Optical Time-Domain Reflectometry}

BOTDR is a coherent detection method that uses a pulsed light. This light is launched into $\mathrm{OF}$ for generating spontaneous BS. As illustrated in Figure 6 [7], back-propagated light is measured with a coherent receiver by mixing the spread signal and local oscillator (LO) signal [11]. Because the back scattered signal power is small, the 
attenuation of the fiber can cause a negative effect on the quality of the measurement. To compensate this disadvantage, the coherent detection mode is used. To extract the local frequency shift, the backscattered signal is optically mixed with the laser signal $\mathrm{CW}$, and the detected pulsed signal is then electrically mixed with the microwave local oscillator signal. The recorded signal is proportional with optical oscillation amplitude at the microwave oscillator frequency, but its dynamic range decreases with the fiber length.

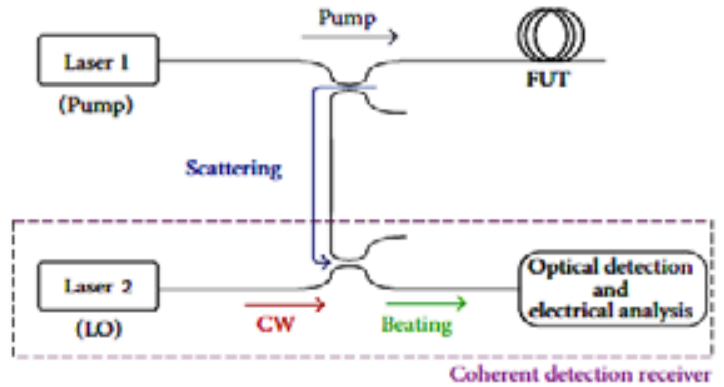

Figure 6. Typical configuration for a BOTDR system. FUT - fiber under test (distributed sensor).

The main disadvantages of this method are: spatial resolution $\geq 1 \mathrm{~m}$; frequency shift is simultaneous dependent by temperature and the longitudinal strait; it is necessary to introduce an electronic filtering to remove the Rayleigh signal.

\subsection{Brillouin Optical frequency-domain analysis}

An alternative approach for the BS shift measurement is BOFDA. This method is based on measuring a complex baseband transfer function relating to the pump counter-propagated waves and the Stokes waves along the fiber amplitude [12]. Such a transfer function is measured for a pumpprobe frequency deviation range, so that, the fiber BFS could be recovered from each location.

An important advantage provided by BOFTA systems in comparison to the BOTDA ones is the operating possibility in narrowband. But a disadvantage of BOFDA is the relatively long measurement time, because it requires multiple measurements at different frequencies to obtain baseband transfer function with a sufficient spectral resolution. During the measurement, OF temperature and strait must be constant not to affect the measurement reliability.

\subsection{Brillouin Optical Correlation-Domain Analysis}

The based approach on optical correlation gave birth to the BOCDA technique, and this is based on the correlation between the probe and pump CW light waves which generate SBS. The correlation based on $\mathrm{CW}$ technique realizes the selectiveposition generating of SBS by controlling the correlation between the light waves of the pump and counter-propagated probe and simultaneously frequency modulation of them by a sine wave which generates the correlation with the periodic peaks [13].

The disadvantage of BOCDA system is limited measuring range due to the periodic nature of the detection position, the measuring length is shorter than other Brillouin sensors, the transducer is more complex and post-processing is more laborious, increasing the measurement time.

\section{Applications and performance}

The applications of sensitive distributed $\mathrm{OF}$ extend from the health monitoring systems to technical structures, in research and medicine (medical instrumentation). They are part of devices for measurement of: temperature and strain, pressure in enclosures, high voltage transformers, electric power lines etc. They can detect: fire, leakage of gases and liquids in pipes, intrusions in perimeters and enclosure, defects in structures etc. They become indispensable in monitoring and diagnostics applications for: means of conveyance (planes, ships, cars); damage from earthquakes; deterioration of large structures (bridges, dams, buildings, tunnels).

The main performance of systems based on Brillouin distributed OF sensitive achieved over the last three years, are shown in Tables 1 and 2.

Table 1. Performance chart of Brillouin distributed fiber sensors based on light backscattering, out of [14].

\begin{tabular}{|l|c|c|c|}
\hline Parameter & BOTDR & BOTDA & $\begin{array}{c}\text { Brillouin } \\
\text { Grating }\end{array}$ \\
\hline Spatial resolution & meter & cm to meter & $\mathrm{cm}$ \\
\hline Sensing range & $\begin{array}{c}\text { Tens } \\
\text { of } \mathrm{km}\end{array}$ & $\begin{array}{c}\text { Up to hun- } \\
\text { dreds of } \mathrm{km}\end{array}$ & $\begin{array}{c}\text { Tens of } \\
\text { meters }\end{array}$ \\
\hline Measurement time & $1-5 \mathrm{~min}$ & $2-5 \mathrm{~min}$ & $+10 \mathrm{~min}$ \\
\hline Temperature, strain & Yes & Yes & Yes \\
\hline Temperature accuracy & $2-3{ }^{\circ} \mathrm{C}$ & $1-2{ }^{\circ} \mathrm{C}$ & $1{ }^{\circ} \mathrm{C}$ \\
\hline Strain accuracy & $60 \mu \varepsilon$ & $20 \mu \varepsilon$ & $10 \mu \varepsilon$ \\
\hline Dynamic measurement & No & $400 \mathrm{~Hz}$ & No \\
\hline
\end{tabular}


Table 2. Performance chart for Brillouin distributed OF sensors, extracted out of [15].

\begin{tabular}{|l|c|c|c|}
\hline \multicolumn{1}{|c|}{ Parameter } & DPP-BOTDA & Brillouin Grating & BOTDR \\
\hline Spatial resolution & $2 \mathrm{~cm}(2 \mathrm{~km}) ; 2 \mathrm{~m}(150 \mathrm{~km})$ & $1-2 \mathrm{~cm}$ & $\sim 1 \mathrm{~m}$ \\
\hline Sensing range & $150-200 \mathrm{~km}$ & $20 \mathrm{~m}$ & $20-50 \mathrm{~km}^{*}$ \\
\hline Measurement time & $2-5 \mathrm{~min}$ & $10+\mathrm{min}$ & $1-5 \mathrm{~min}$ \\
\hline Temperature and strain & Yes & Yes & Yes \\
\hline Temperature accuracy & $1-2{ }^{\circ} \mathrm{C}$ & $1{ }^{\circ} \mathrm{C}$ & $2-3^{\circ} \mathrm{C}$ \\
\hline Strain accuracy & $20 \mu \varepsilon$ & No & $60 \mu \varepsilon$ \\
\hline Dynamic measurement & yes & $* *$ & No \\
\hline Calibration & $* *$ & $* *$ \\
\hline $\begin{array}{l}\text { Light source } \\
\text { requirement }\end{array}$ & $\begin{array}{c}\text { Two DFB lasers (frequency locking } \\
\text { of two lasers is required) }\end{array}$ & $\begin{array}{c}\text { Three lasers (frequency locking } \\
\text { of two lasers is required) }\end{array}$ & $\begin{array}{c}\text { One narrow } \\
\text { linewidth laser }\end{array}$ \\
\hline Detectors & Broadband & Broadband & High sensitivity \\
\hline Detection scheme & Direct & Direct & Coherent \\
\hline
\end{tabular}

* - Presented by Yokogawa Electric Corporation; ** - Determined by fiber property.

\section{Conclusions}

Performances of different techniques and methods are markedly different, each of them can be used according to specific application types.

Performances obtained until now have allowed the realization of functional equipment and systems in practical activity, but studies aim at the methods and techniques diversifying, including a combination of Brillouin, Raman and Rayleigh effects in order to improve the performance and diversify the applications.

\section{Acknowledgement}

This work was supported by Executive Agency for Higher Education, Research, Development and Innovation Funding and by Romanian Ministry of Structural Funds, under projects ENIAC 04/2014 as well as FOSLAB.

\section{References}

1) Still T, High Frequency Acoustics in Colloid-Based Meso and Nanostructures by Spontaneus Brillouin Light Scattering, Springer Hardcover, 2010

2) Aldo Minardo, Fiber-optic distributed straintemperature sensors based on stimulated Brillouin scattering, PhD Thesis, Seconda Universita' Degli Studi di Napoli, 2004

3) Anatoli A. Chtcherbakov, Pieter L. Swart, and Stephanus J. Spammer, Mach-Zehnder and modified Sagnac-distributed fiber-optic impact sensor, APPLIED OPTICS, Vol. 37, No. 16, (1998), p. 3432

4) Luc Thévenaz, Brillouin dynamic grating distributed sensing (BDG-DS), Brillouin distributed time-domain sensing in optical fibers: state of the art and perspectives, Front. Optoelectron. China 2010,3(1): 13-21
5) C. Wang and K. Shida, A novel multifunctional distributed optical fiber sensor based on attenuation, Proc. IMTC'06, pp. 2018-2023, April, 2006

6) Anbhawa Nand, Distributed Fibre Bragg Grating based Sensors, Integrated sensing using chirped optical fiber gratings, $\mathrm{PhD}$ Thesis, Victoria University Australia, 2007

7) C. A. Galindez-Jamioy and J. M. Lopez-Higuera, Brillouin Distributed Fiber Sensors: An Overview and Applications, Hindawi Publishing Corporation, Journal of Sensors, Volume 2012, Article ID 204121,17pages, doi:10.1155/2012/204121.

8) A. Zornoza, R.Perez-Herrera, C.Elosua et al., "Longrange hybrid network with point and distributed Brillouin sensors using Raman amplification," Optics Express, vol. 18, no. 9, pp. 9531-9541, 2010.

9) W.Li, X.Bao, Y.Li, and L.Chen, "Differential pulsewidth pair BOTDA for high spatial resolution sensing" Optics Express, vol.16, no.26, pp.21616-21625, 2008.

10) K. Y. Song, "Operation of Brillouin dynamic grating in single mode optical fibers," Optics Letters, vol. 36, no. 23, pp. 4686-4688, 2011.

11) Y. Lu, H. Liang, X. Zhang, and F. Wang, Brillouin optical time-domain reflectometry based on Hadamard sequence probe pulse, Proceedings, the $9^{\text {th }}$ International Conference on Optical Communications and Networks (ICOCN '10), pp. 36-38, October 2010.

12) D. Garus, T. Gogolla, K. Krebber, and F. Schliep, "Brillouin optical-fiber frequency-domain analysis for distributed temperature and strain measurements", J. Lightwave Technol., vol. 15, pp. 654-662, 1997.

13) K.Hotate and M.Tanaka, "Distributed fiber Brillouin strain sensing with a 1-cm spatial resolution by correlation-based continuous-wave technique", IEEE Phton. Technol. Lett., vol.14, no.2, pp.179-181, 2002.

14) Zengguang Qin, Distributed Optical Fiber Vibration Sensor Based on Rayleigh Backscattering, PhD Thesis, University of Ottawa, Canada, 2013.

15) X. Bao, L. Chen, Recent progress in distributed fiber optic sensors,Sensors,vol.12, no.7,pp.8601-8639,2012. 